\title{
Fluid Extensions for Optical Flow and Diffusion-based Image Registration
}

\author{
Jens-Peer Kuska ${ }^{1}$, Patrick Scheibe ${ }^{2}$, Ulf-Dietrich Braumann ${ }^{2}$ \\ ${ }^{1}$ Interdisciplinary Centre for Bioinformatics, University Leipzig, 04107 Leipzig \\ ${ }^{2}$ Translational Centre for Regenerative Medicine, University Leipzig, 04103 Leipzig \\ kuska@informatik.uni-leipzig.de
}

\begin{abstract}
The present paper derives two new equations for the nonlinear registration of images based on Euler-Lagrange equations. It offers a systematic way to construct fluid extensions to registration equations based on variational principles.
\end{abstract}

\section{Introduction}

Nonlinear image registration has various applications in image processing ranging from three-dimensional reconstruction of serial sections over segmentation with help of image atlases to the measurement of changes during the development of a disease.

The goal of image registration is to find a vector transformation $\boldsymbol{u}(\boldsymbol{x})$ so that the sample image $S(\boldsymbol{x})$ under the transformation $S(\boldsymbol{x}-\boldsymbol{u}(\boldsymbol{x}))$ matches the template image $T(\boldsymbol{x})$. Additionally, such transformation $\boldsymbol{u}(\boldsymbol{x})$ is required to satisfy some smoothness condition. One way to obtain such a transformation is to use physically motivated equations, e.g. the Navier-Lamé equation for the deformation $\boldsymbol{u}$ [1] or solving the Navier-Lamé equation for the velocity field $\dot{\boldsymbol{u}}$ and to use the time integral of the velocity as the displacement field [2]. The variant that uses the two fields $\dot{\boldsymbol{u}}(\boldsymbol{x})$ and $\boldsymbol{u}(\boldsymbol{x})$ is called fluid registration.

Another possibility to derive a differential equation for the displacement field is to apply a variational approach $[3,4,5,6]$ that combines various conditions for the displacement field. This approach typically yields a static, time and velocity independent equation for the displacement field $\boldsymbol{u}(\boldsymbol{x})$. To obtain a smooth convergence of the nonlinear equation one introduces an artificial time and solves a diffusion-like equation $[7,8]$. Since the displacement field is modified by a diffusion like process, the convergence of the variational equations is rather slow comparing to the fluid registration. The present paper offers a way to construct dynamic equations for the velocity field on the basis of variational equations without the help of fluid dynamics.

\section{Materials and Methods}

\subsection{Undamped Equation of Motion}

For the registration of the sample image $S(\boldsymbol{x})$ onto the template image $T(\boldsymbol{x})$ with $\boldsymbol{x} \in \mathbb{R}^{2}$, a displacement field $\boldsymbol{u}(\boldsymbol{x}, t)=\left(u_{1}(\boldsymbol{x}, t), u_{2}(\boldsymbol{x}, t)\right)^{\top}$ with $\left\{\boldsymbol{u}: \mathbb{R}^{2} \mapsto \mathbb{R}^{2}\right\}$ 
must be found so that

$$
\mathcal{V}[\boldsymbol{u}]=\frac{1}{2} \int_{\Omega}(T(\boldsymbol{x})-S(\boldsymbol{x}-\boldsymbol{u}(\boldsymbol{x}), t))^{2} \mathrm{~d}^{2} \boldsymbol{x}
$$

should be minimal. The displacement field should obey some smoothness condition. We will use the function $\mathcal{T}[\dot{\boldsymbol{u}}]$ for the smoothness of the Lagrangian. For the case of optical flow-based image registration the smoothness constraint is defined as

$$
\mathcal{T}_{\mathrm{F}}[\dot{\boldsymbol{u}}]=\frac{\mu}{2} \int_{\Omega} \sum_{i=1}^{2}\left(\Delta \dot{u}_{i}(\boldsymbol{x}, t)\right)^{2} \mathrm{~d}^{2} \boldsymbol{x}
$$

and for the diffusion registration

$$
\mathcal{T}_{\mathrm{D}}[\dot{\boldsymbol{u}}]=-\frac{\mu}{2} \int_{\Omega} \sum_{i=1}^{2}\left(\nabla \dot{u}_{i}(\boldsymbol{x}, t)\right)^{2} \mathrm{~d}^{2} \boldsymbol{x}
$$

The Euler-Lagrange equation for

$$
\mathcal{L}(\dot{\boldsymbol{u}}, \boldsymbol{u})=\mathcal{T}[\dot{\boldsymbol{u}}]-\mathcal{V}[\boldsymbol{u}]
$$

yields

$$
\mu \frac{\mathrm{d}^{2}}{\mathrm{~d} t^{2}}\left(\Delta^{2} \boldsymbol{u}\right)+[T(\boldsymbol{x})-S(\boldsymbol{x}-\boldsymbol{u}(\boldsymbol{x}, t))] \nabla S(\boldsymbol{x}-\boldsymbol{u}(\boldsymbol{x}, t)=0
$$

for the optical flow-based image registration and

$$
\mu \frac{\mathrm{d}^{2}}{\mathrm{~d} t^{2}}(-\Delta \boldsymbol{u})+[T(\boldsymbol{x})-S(\boldsymbol{x}-\boldsymbol{u}(\boldsymbol{x}, t))] \nabla S(\boldsymbol{x}-\boldsymbol{u}(\boldsymbol{x}, t)=0
$$

for the diffusion registration. Both equations are free from dissipation of the energy $\mathcal{H}=\mathcal{T}+\mathcal{V}$ and the solution will start to oscillate. Since we are interested in the minimum of $\mathcal{V}$ one has to add friction forces.

\subsection{Dissipative Forces}

Friction forces are not an integral part of the Euler-Lagrange equations but they can be included as additional summand in the force Eqns. (5) and (6), respectively. We will add two kinds of friction forces, one that act independently of the position in space, and a second force that includes the spatial dependence by space derivatives of the velocity field. As position-independent friction force the function $\boldsymbol{f}_{1}=-\mu \gamma \dot{\boldsymbol{u}}$ will be used. The second dissipative force will depend on the partial derivatives of the velocity field. The Navier-Stokes equation would suggest a term proportional to $\boldsymbol{f}_{\mathrm{v}}=-\mu \nu \Delta \dot{\boldsymbol{u}}$ (the viscosity term), and for the optical flow-based case a term $\boldsymbol{f}_{\mathrm{v}}=-\mu \nu \Delta^{2} \dot{\boldsymbol{u}}$ will be used.

Adding the two friction forces to the left hand side of the Eqns. (5) and (6), the fluid extension for the optical flow-based registration is given by the solution of the equation

$$
\begin{gathered}
\mu \frac{\mathrm{d}^{2}}{\mathrm{~d} t^{2}}\left(\Delta^{2} \boldsymbol{u}\right)+[T(\boldsymbol{x})-S(\boldsymbol{x}-\boldsymbol{u}(\boldsymbol{x}, t))] \nabla S(\boldsymbol{x}-\boldsymbol{u}(\boldsymbol{x}, t))= \\
-\mu \gamma \dot{\boldsymbol{u}}(\boldsymbol{x}, t)-\mu \nu \Delta^{2} \dot{\boldsymbol{u}}(\boldsymbol{x}, t)
\end{gathered}
$$


and for the diffusion-based version one gets

$$
\begin{gathered}
\mu \frac{\mathrm{d}^{2}}{\mathrm{~d} t^{2}}(-\Delta \boldsymbol{u})+[T(\boldsymbol{x})-S(\boldsymbol{x}-\boldsymbol{u}(\boldsymbol{x}, t))] \nabla S(\boldsymbol{x}-\boldsymbol{u}(\boldsymbol{x}, t))= \\
-\mu \gamma \dot{\boldsymbol{u}}(\boldsymbol{x}, t)-\mu \nu \Delta \dot{\boldsymbol{u}}(\boldsymbol{x}, t) .
\end{gathered}
$$

The only force free solution of the equations is obtained for the case that the displacement field $\lim _{t \rightarrow \infty} \boldsymbol{u}(\boldsymbol{x}, t)$ transforms the sample image on the template image. In this case, the force $[T(\boldsymbol{x})-S(\boldsymbol{x}-\boldsymbol{u}(\boldsymbol{x}, t))] \nabla S(\boldsymbol{x}-\boldsymbol{u}(\boldsymbol{x}, t))$ is zero, and the friction will cause the velocity field also going to zero. The differential constraint to the velocity field may hinder a perfect match in this case, the vector fields will oscillate, but the friction term $\boldsymbol{f}_{\mathrm{v}}$ hinders the creation of waves thus keeping oscillations local. A sufficiently strong damping should restrict the local oscillation amplitude to values smaller than the pixel distance.

With the two Green functions

$$
\begin{aligned}
G_{\mathrm{F}}\left[\Delta^{2} g(\boldsymbol{x})\right] & =g(\boldsymbol{x}) \\
G_{\mathrm{D}}[(-\Delta) g(\boldsymbol{x})] & =g(\boldsymbol{x})
\end{aligned}
$$

one gets the explicit system of second order equations that can be solved by a standard method for initial value problems. With either $G=G_{\mathrm{F}}$ or $G=G_{\mathrm{D}}$ the explicit version of the equations (7) and (8) can be written as

$$
\begin{aligned}
\ddot{\boldsymbol{u}}(\boldsymbol{x}, t)=- & \frac{1}{\mu} G[(T(\boldsymbol{x})-S(\boldsymbol{x}-\boldsymbol{u}(\boldsymbol{x}, t))) \nabla S(\boldsymbol{x}-\boldsymbol{u}(\boldsymbol{x}, t))] \\
& -\gamma G[\dot{\boldsymbol{u}}(\boldsymbol{x}, t)]-\nu \dot{\boldsymbol{u}}(\boldsymbol{x}, t) .
\end{aligned}
$$

\section{$3 \quad$ Results}

Three examples of the fluid extension of diffusion and optical flow-based registration will be shown. The integration of the equations of motion is stopped when the registered image $S(\boldsymbol{x}-\boldsymbol{u}(\boldsymbol{x}))$ does not change more than $1 \times 10^{-5}$ per pixel. The intensities of sample and template image are all normalized to the interval $[0,1]$. The step size control typically takes hundred times larger time steps for the diffusion-based registration than for the optical flow-based variant, but also requires longer absolute time intervals to achieve convergence.

The first example maps a circle on a $128 \times 128$ grid onto the letter "C" (Fig. 1a). The parameters for the optical flow-based registration are $(\mu, \nu, \gamma)=$ $(0.5,0.00045,8)$ and for the diffusion-based equation $(\mu, \nu, \gamma)=(0.125,0.00045,8)$.

As a second example we show the transformation of an angry face onto a smiling one (Fig. 1b). This example in particular is challenging for the registration because the vector field must develop several singularities to transform the eyebrows and the mouth. To make the difference between the two displacement fields more illustrative, the distortion of a regular grid as caused by the displacement field is shown as overlay on the registration results. The parameters for the 

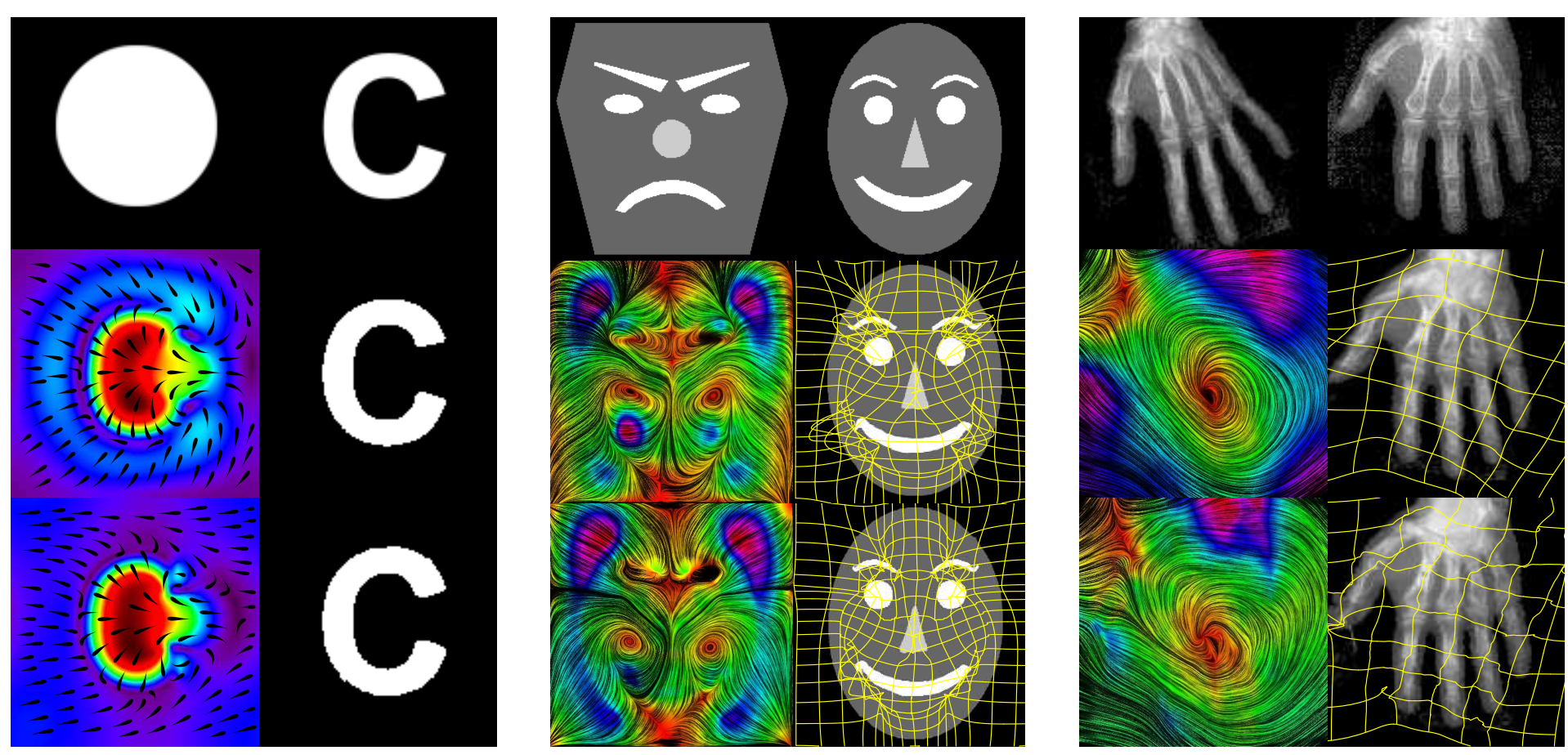

Fig. 1. Overview on three different registration tasks (top row: the image pairs; middle row: results for the fluid extension of the optical flow-based method using Eqn. (7); bottom row: results for the fluid extension of the diffusion-based method using Eqn. (8)). A: Registration of a circle onto the letter "C". B: Registration of an angry "face" onto a smiling one. C: Registration of two radiographs of different hands. The respective displacement vector fields are To underline the structure of the singularities in the respective displacement vector fields $\boldsymbol{u}(\boldsymbol{x})$, a line integral convolution [9] is used to visualize them (a: with flow fishes, b/c: with dot patterns, underlying color codes displacement magnitude) 
optical flow-based version are $(\mu, \nu, \gamma)=(0.5,0.00015,6)$ and for the diffusion registration $(\mu, \nu, \gamma)=(0.5,0.0003,6)$.

The last example maps two radiographs of hands (Fig. 1c). The overlayed displacement of the regular mesh shows the lower smoothness of the diffusion registration compared with the optical flow-based variant.

\section{Discussion}

We have presented two new equations for nonlinear image registration based on the optical flow and the diffusion registration. Instead of the introduction of an artificial time, the new equations require a smooth velocity field and solve a system of second order differential equations for the displacement field. Both registration methods can be treated in a unified way. Based on the underlying variational approach, the design of special fluid like registration equations is possible by addition of future constrains for the displacement or the velocity field.

Acknowledgement. The project is supported by the Federal Ministry of Education and Research (BMBF), grant PTJ-BIO/31P4282 (MS CartPro) and grant PTJ-BIO/031390 (TRM Leipzig).

\section{References}

1. Christensen GE, Rabbit RD, Miller MI. Deformable templates using large deformation kinematics. IEEE Trans Image Process. 1996;10(5):1435-47.

2. Bro-Nielsen M, Gramkow C. Fast fluid registration of medical images. Proc VBC. 1996; p. 267-76.

3. Braumann UD, Kuska JP. A new equation for nonlinear image registration with control over the vortex structure in the displacement field. Proc ICIP. 2006; p. 329-32.

4. Amit Y. A nonlinear variational problem for image matching. SIAM J Sci Comp. 1994;15(1):207-24.

5. Modersitzki J. Numerical Methods for Image Registration. New York, NY: Oxford University Press; 2004.

6. Fischer B, Modersitzki J. A unified approach to fast image registration and a new curvature based registration technique. Linear Algebra Appl. 2004;380:107-24.

7. Fischer B, Modersitzki J. Fast diffusion registration. AMS Contemporary Mathematics, Inverse Problems, Image Analysis, and Medical Imaging. 2002;313:117-29.

8. Fischer B, Modersitzki J. Curvature based image registration. J Math Imaging Vis. 2003;18(1):81-5.

9. Cabral B, Leedom LC. Imaging vector fields using line integral convolution. Proc SIGGRAPH. 1993; p. 263-72. 